\title{
Combined Pars Plana Vitrectomy and Segmental Scleral Buckle for Rhegmatogenous Retinal Detachment with Inferior Retinal Breaks
}

\author{
Parnian Arjmand ${ }^{1,2}$ \\ Tina Felfeli (iD ${ }^{1,3}$ \\ Efrem D Mandelcorn ${ }^{1,2}$ \\ 'Department of Ophthalmology and \\ Vision Sciences, University of Toronto, \\ Toronto, Ontario, Canada; ${ }^{2}$ Department \\ of Ophthalmology, Toronto Western \\ Hospital, University Health Network, \\ Toronto, Ontario, Canada; ${ }^{3}$ Institute of \\ Health Policy, Management and \\ Evaluation, Dalla Lana School of Public \\ Health, University of Toronto, Toronto, \\ Ontario, Canada
}

Correspondence: Efrem D Mandelcorn Department of Ophthalmology, Toronto Western Hospital, University Health

Network, 6E-432, 399 Bathurst St,

Toronto, ON, M5T2S8, Canada

Tel + 4166035418

Fax +I 4166035193

Email efrem.mandelcorn@utoronto.ca
Purpose: To describe a variation of the traditional segmental scleral buckle (SB) without an encircling band combined with 23-gauge pars plana vitrectomy (PPV) for the management of rhegmatogenous retinal detachment (RRD) with inferior retinal breaks.

Patients and Methods: This is a single-center, retrospective, consecutive review of all RRDs with inferior retinal breaks that were treated with PPV and segmental SB without an encircling band between May 2019 and February 2020.

Results: A total of 12 eyes of 12 patients were included in the study. All patients had at least 1 inferior retinal break and more than 2 clock hours of retinal detachment. Eight eyes had RRD with macular involvement at presentation. Seven eyes had a persistent RRD following previous pneumatic retinopexy (C3F8). All eyes were treated by PPV combined with a segmental \#510 sponge without an encircling band. Surgery anatomical success was $100 \%$. Mean $\operatorname{logMAR}$ visual acuity was 1 (SD $0.6 ; 20 / 160)$ and 0.5 (SD $0.4 ; 20 / 60$ ) at 3 months and last follow-ups, respectively. No scleral buckle-related complications were noted over the 4.1 (SD 0.8) month follow-up period.

Conclusion: The combined segmental buckling technique is a safe and effective adjunct to PPV in treatment of inferior RRD.

Keywords: retinal breaks, vitreoretinal surgery, outcomes, surgical technique, retina, retrospective study

\section{Introduction}

Pars plana vitrectomy (PPV), scleral buckle (SB), or a combination of both have been described in the management of rhegmatogenous retinal detachment (RRD) secondary to inferior retinal breaks. RRDs with inferior breaks generally confer a higher risk of recurrent detachment. This is often due to the lack of adequate gas or silicone oil tamponade to the inferior retina, challenging patient posturing, as well as factors such as higher risk of PVR and missed breaks due to vitreous hemorrhage.$^{1-3}$ To date, the surgical procedure of choice including PPV versus SB, the use of gas versus silicone oil, heavy silicone oil, drainage technique, the extent of laser, and post-operative positioning remain controversial. ${ }^{1-4}$

In the era of small-gauge vitrectomy and wide-angle viewing systems, there has been a shift away from scleral buckling in consideration of the longer operative time and post-operative patient discomfort, diplopia and axial length changes associated with SB. ${ }^{1,3-5}$ Nevertheless, it has been demonstrated that in cases of multiple anterior or inferior breaks or $\mathrm{RD}$ with proliferative vitreoretinopathy 
(PVR), combining an encircling scleral buckle with PPV may improve anatomical outcomes. ${ }^{1-6}$ The Single surgery success rate of combined PPV/SB has been reported to be significantly higher in eyes with inferior breaks compared to PPV alone in phakic or pseudophakic eyes in recent large cohort studies. ${ }^{1,6,7}$ Nevertheless, several studies have demonstrated comparable outcomes in these cases with PPV alone, especially in pseudophakic eyes, while avoiding the potential complications associated with the presence of an encircling band. ${ }^{6,8}$

Many variations of scleral buckling with or without PPV have been described in the literature. ${ }^{9,10}$ Most attempt at minimizing complications, without affecting the added benefits of buckling support. Scleral buckles with an encircling band may be complicated by retinal perforation, hypotony, anterior segment ischemia, choroidal hemorrhage, and significant axial myopia, diplopia and asymmetry. ${ }^{1,9}$ Segmental buckles eliminate the risk of radial retinal folds, offer a lower risk of fish-mouthing of the retinal break, and result in minimal axial lengthening. ${ }^{10-12}$ To date, the combination of segmental SB and PPV has not been described.

Herein, we present a case series of patients who underwent combined segmental buckling, without an encircling band, with 23-gauge PPV for the management of RRDs secondary to inferior retinal breaks.

\section{Materials and Methods}

A retrospective review of all consecutive cases with RRD with at least one inferior retinal break who underwent surgical repair with 23-gauge PPV and SB combination between May 2019 and February 2020 by a single vitreoretinal surgeon at a tertiary care academic institution, Toronto Western Hospital, Kensington Eye Institute, Toronto, Ontario was conducted. Patients were excluded if they had less than 3 months of follow-up, were treated with PPV or SB alone, received an encircling SB band or were previously treated with a SB or PPV in the study eye. Institutional ethics review board was obtained from Toronto Western Hospital, University Health Network. Given the retrospective nature of the study and lack of feasibility for contacting all patients, requirement for patient consent was waived by the ethics committee given the retrospective nature of the study. This study adhered to patient data confidentiality guidelines and compliance with the Declaration of Helsinki.

Data including age, chronicity of RRD, pre-operative and post-operative visual acuity, lens status, extend of $\mathrm{RD}$, number and type of retinal breaks, macular status and presence of vitreous hemorrhage ( $\mathrm{VH}$, defined as large $\mathrm{VH}$ posterior to the equator or 5 clock hours central or peripheral $\mathrm{VH})$, type and number of scleral buckle segments, choice of tamponade agent, and intra- or post-operative complications were collected. The primary measured outcome was surgery anatomical success. Secondary measured outcome was post-operative PVR and visual acuity at 3-month and final follow-up.

Patient clinical characteristics, surgical details and outcomes were reported descriptively. Snellen visual acuity was converted to logarithm of the minimum angle of resolution $(\log$ MAR) values. The $\log$ MAR values for visual acuity of "light perception", "hand motion" and "counting fingers" were assigned 2.7, 2.2 and 1.7, respectively, based on previously published literature. ${ }^{13}$

\section{Surgical Technique}

Vitrectomy was performed using a standard 3-port 23gauge PPV using a noncontact wide-angle viewing system (Resight 700, Carl Zeiss Meditech; Oberkochen, Germany, or Binocular Indirect Ophthalmomicroscope; Oculus, Wetzlar, Germany). Endolaser photocoagulation was applied around the retinal tear or 360 degrees to the vitreous base. In all cases, patients received perfluoron-octane (C3F8, 15-18\%), sulphur hexafluoride (SF6, $25 \%$ ), Densiron ${ }^{\circledR}$ Xtra, or Siluron ${ }^{\circledR} 2000$.

The location of the inferior-most break was marked externally using a scleral depressor marker under direct visualization with the Resight. A partial inferior peritomy was then performed around the inferior rectus muscle (2-3 clock hours). For breaks located between 5 and 7 clock hours, only the inferior rectus muscle was isolated with a single $2-0$ silk tie. For inferior breaks between 3 to 5 and 7 to 9 clock hours, one horizontal muscle as well as the inferior rectus muscle were each isolated with a $2-0$ silk tie. Horizontal mattress suture(s) were placed using 5-0 Nylon in the lower quadrant(s) (Figure 1). A segmental scleral buckle (\#510 silicone sponge, FCI-ophthalmics, Pembroke, MA, USA) was passed under the marked break(s) and secured with the suture (Video.mp4 Supplemental Digital Content 1).

\section{Results}

A total of 12 eyes of 12 patients with a mean age of 66.8 (SD 12.8) underwent the combined segmental buckling technique. Twenty percent of patients were female. All patients had at least one inferior retinal break (range 1- 


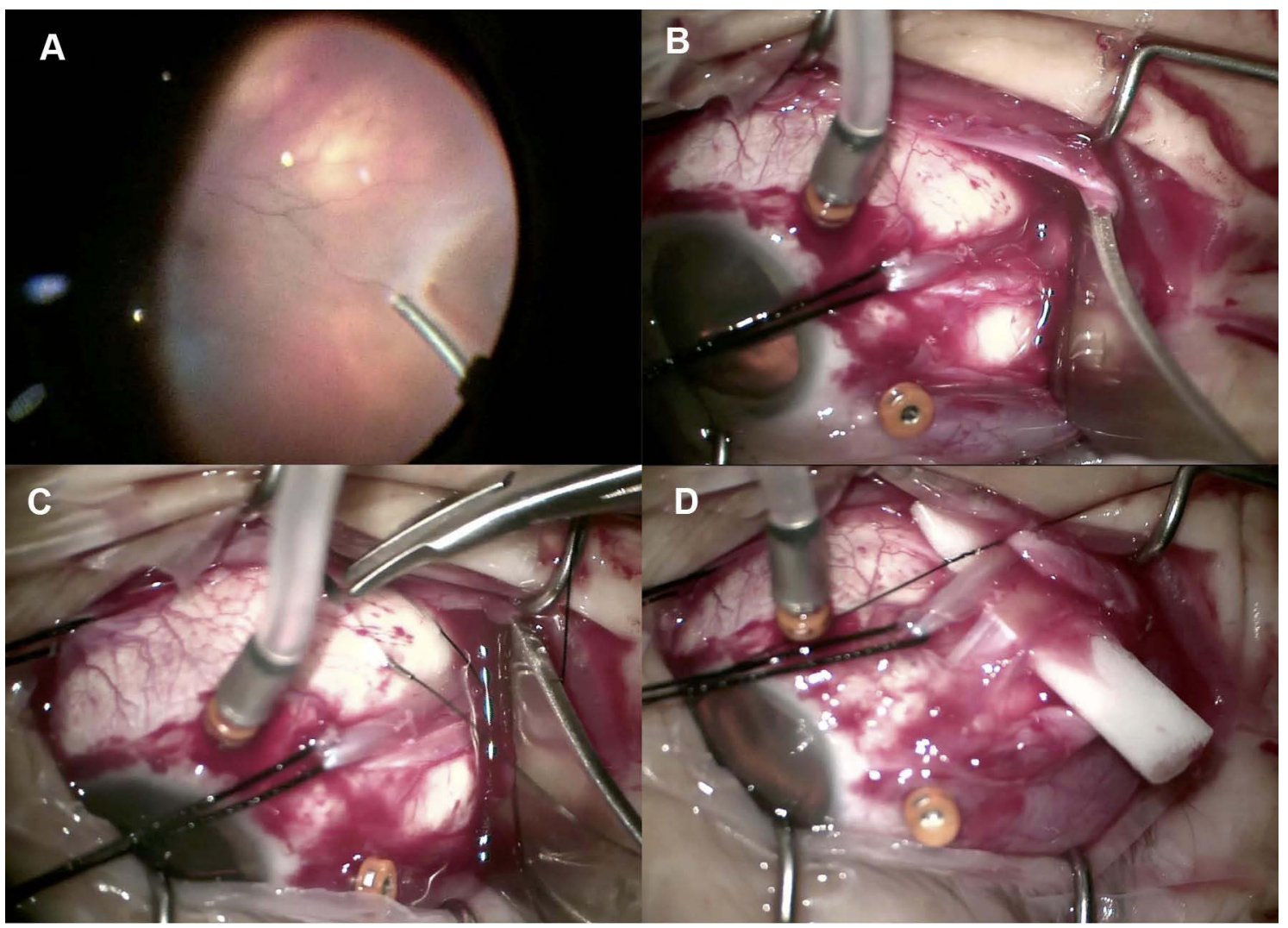

Figure I The location of the inferior-most break is marked externally using a scleral depressor marker under direct visualization (A). A partial inferior peritomy around the inferior rectus muscle is then performed and a single 2-0 silk tie is used to isolate an inferior rectus muscle with or without a horizontal muscle based on the location of the break(s) (B). Horizontal mattress sutures are placed using 5-0 Nylon in the lower quadrant (C). A segmental scleral buckle is then passed under the marked break and secured with the suture $(\mathbf{D})$.

3), while 9 eyes also had superior breaks (range 1-3). The mean extent of RD was 6 (SD 2.1) clock hours. Only two eyes had vitreous hemorrhage and one eye had evidence of PVR grade B pre-operatively. Eight eyes had macular involvement at presentation. Seven eyes had a persistent RRD following previous pneumatic retinopexy (C3F8). The mean duration of visual symptoms was 10.6 days (SD 9.8) and none of the RD's had chronic features on presentation. C3F8 gas (18\%) was used in the majority of cases, followed by SF6, Siluron 2000 (Labtician Ophthalmics, Oakville, Canada), and Densiron XTRA (Fluoron GmbH, Germany) (Table 1).

There were no intraoperative complications. Single surgery anatomical success was $100 \%$ at the last followup. The mean visual acuity at baseline, 3 months and at last follow-up were 1.4 (SD 0.9; 20/400), 1 (SD 0.6; 20/ 160 ) and 0.5 (SD $0.4 ; 20 / 60$ ), respectively (Table 2 ). Only 1 patient had grade B PVR on presentation, which resolved without re-detachment at the final follow-up.

None of the repairs required additional intravitreal gas injection. All 12 eyes had good buckle height post- operatively. One eye developed epiretinal membrane and one eye developed cystoid macular edema postoperatively. None of the patients developed strabismus, implant extrusion or retinal folds. No other scleral bucklerelated complications were noticed at last follow-up. The mean follow-up duration was 4.1 (SD 0.8) months.

\section{Discussion}

Retinal detachments with inferior breaks jeopardize single surgery success rates due to the potential for incomplete tamponade and higher risk of PVR. ${ }^{1-3,6-8}$ The recent PRO study (report number 9) comparing anatomical success in eyes of patients with primary RD and a single inferior break found a significant advantage for PPV/SB (with an encircling band) (87.4\%) vs PPV alone (76.8\%) at 1-year follow-up. ${ }^{6}$ Other recent studies, with different tamponade agents and lower than average success rates, have reported comparable outcomes with PPV vs PPV/SB alone..$^{6-8,14,15}$ Perhaps, the main caveat inherent to any retinal detachment study is the presence of variables which cannot always be well-accounted for which significantly 
Table I Baseline Demographics and Ocular Characteristics of Patients with Inferior Rhegmatogenous Retinal Detachment (RD)

\begin{tabular}{|l|l|l|}
\hline & & SD \\
\hline Age & 66.8 & \\
Female & $3(25 \%)$ & \\
Pseudophakic & $6(50 \%)$ & \\
Avg \# of clock hours of RD & 6 & 2.12 \\
\# of retinal breaks & 3.41 & 1.83 \\
\# of inferior breaks & 1.5 & 0.9 \\
\# of superior breaks & 1.41 & 1.16 \\
Patients with a total RD & $0(0 \%)$ & \\
Vitreous hemorrhage & $2(16.6 \%)$ & \\
Macular detachment & $8(66.6)$ & \\
Avg duration of RD (days) & 10.58 & 9.82 \\
PVR & $1(8.3 \%)$ & \\
Pre-operative intravitreal gas & $7(58 \%)$ & \\
\hline Choice of tamponade agent & & \\
SF6 (25\%) & $4(33 \%)$ & \\
C3F8 (I8\%) & $6(50 \%)$ & \\
Siluron & $1(8.3 \%)$ & \\
Densiron & $1(8.3 \%)$ & \\
\hline Complications & & \\
CME & $1(8.3 \%)$ & \\
ERM & $1(8.3 \%)$ & \\
Cataract & $1(8.3 \%)$ & \\
\hline
\end{tabular}

Abbreviations: Avg, Average; \#, Number; PVR, Proliferative vitreoretinopathy; CME, Cystoid macular edema; ERM, Epiretinal membrane; SD, Standard deviation.

contribute to success rates such as: surgeon expertise, accuracy of pre- and intra-operative scleral depressed examination, and patient compliance.

The combined segmental buckling technique offers retinal surgeons a good option when faced with iatrogenic or missed inferior pathology intraoperatively. There are many instances in which inferior breaks may be missed pre-operatively due to media opacity, vitreous hemorrhage or poor patient compliance with a thorough scleral depressed exam. ${ }^{1,4}$ There are also instances in which iatrogenic retinal breaks develop during peripheral vitreous dissection. In these cases, surgeons must choose the appropriate tamponade to avoid reattachment failure by way of using expansile gas with strict head positioning or heavy silicone oil (Densiron ${ }^{\circledR}$ ), which offers an excellent success rate, but which may not be available. ${ }^{3}$ The alternative to these options is a scleral buckle but placing an encircling band after the PPV has already been initiated which can be technically challenging. In most cases of combined PPV and SB, the encircling band is placed at the beginning of the case to ameliorate this technical challenge.

In this study, we demonstrate $100 \%$ anatomical success in the treatment of RRDs with inferior breaks using a minimally invasive scleral buckling procedure without an encircling band. Though the use of segmental sponges as a primary buckling technique was first described over 60 years ago, ${ }^{6}$ 23-gauge PPV with segmental sponges without an encircling band or a circumferential element has not been previously published.

The advantages of this technique are multi-fold, including the good anatomical success rate. None of our patients developed any complications such as strabismus, implant extrusion or retinal folds which are common secondary adverse events with the use of an encircling band. Placing a single segmental SB is also simple and efficient and relatively easy to do even after the PPV has been started since it is minimally invasive when compared to an encircling band. Placing a segmental sponge after PPV without an enriching band allows for a small peritomy and loops around only one or two rectus muscles. It also offers the benefit of excellent visualization of the breaks with the microscope viewing system (in our case the Resight 700) for accurate localization and buckle placement.

This case series is retrospective in nature, and includes a small number of eyes with a relatively short follow-up period. There is also some variability between cases with regards to the tamponade agents used (short- and long-term gas or oil), number of inferior breaks and patient compliance with post-operative positioning. Nonetheless, the heterogeneity of the cases demonstrates the general applicability of this technique. Though this technique is more efficient and less invasive, we did not administer a formal pain survey to patients, nor did we record actual surgical time.

Table 2 Primary and Secondary Outcomes, Namely, Single Surgery Anatomical Success (SSAS) and Visual Acuity (Logarithm of Minimum Angle of Resolution, LogMAR) at Baseline, 3 Months and Last Follow-Up

\begin{tabular}{|l|l|l|l|}
\hline Outcomes & Baseline & 3 Months & Last Follow Up \\
\hline SSAS & & $100 \%$ & $100 \%$ \\
\hline Visual acuity (logMAR) & $1.37 \pm 0.91$ & $0.96 \pm 0.59$ & $0.52 \pm 0.36$ \\
\hline
\end{tabular}

Note: Average time to follow up was 5.6 months. 
A comparative prospective study examining the success rate of PPV alone versus PPV with a segmental buckle will further shed light on the added benefit of segmental support for inferior breaks.

\section{Conclusion}

Overall, the placement of a segmental SB is a safe and effective technique to enhance anatomical surgical success and offers vitreoretinal surgeons a good option when encountering inferior pathology during PPV for RRD.

\section{Disclosure}

Dr Efrem D Mandelcorn received Speaker honorarium from Bayer and Novartis, outside the submitted work. The author reports no other conflicts of interest in this work.

\section{References}

1. Storey P, Alshareef R, Khuthaila M, et al. Pars plana vitrectomy and scleral buckle versus pars plana vitrectomy alone for patients with rhegmatogenous retinal detachment at high risk for proliferative vitreoretinopathy. Retina. 2014;34(10):1945-1951. doi:10.1097/ IAE.0000000000000216

2. Totsuka K, Inui H, Roggia MF, Hirasawa K, Noda Y, Ueta T. supplemental scleral buckle in vitrectomy for the repair of rhegmatogenous retinal detachment. Retina. 2015;35:2423-2431. doi:10.1097/ IAE.0000000000000797

3. Hostovsky A, Yap J, Mandelcorn MS, Mandelcorn ED. Densiron ${ }^{\circledR} 68$ heavy silicone oil as a short-term intraocular tamponade for macula-on inferior retinal detachments - a case series. Retin Cases Brief Rep. 2020. doi:10.1097/ICB.0000000000001037

4. Wickham L, Connor M, Aylward GW. Vitrectomy and gas for inferior break retinal detachments: are the results comparable to vitrectomy, gas, and scleral buckle? Br J Ophthalmol. 2004;88(11):1376-1379. doi:10.1136/bjo.2004.043687
5. Kreissig I, Rose D, Jost B. Minimized surgery for retinal detachments with segmental buckling and nondrainage. An 11-year follow-up. Retina. 1992;12(3):224-231.

6. Starr MR, Obeid A, Ryan EH, et al. Retinal detachment with inferior retinal breaks: primary vitrectomy versus vitrectomy with scleral buckle(Pro study report no. 9). Retina. 2021;41(3):525-530. doi:10.1097/IAE.0000000000002917

7. Joseph DP, Ryan EH, Ryan CM, et al. Primary retinal detachment outcomes study: pseudophakic retinal detachment outcomes: primary retinal detachment outcomes study report number 3. Ophthalmology. 2020;127(11):1507-1514. doi:10.1016/j.ophtha.2020.05.005

8. Martínez-Castillo VJ, García-Arumí J, Boixadera A. Pars plana vitrectomy alone for the management of pseudophakic rhegmatogenous retinal detachment with only inferior breaks. Ophthalmology. 2016;123:1563-1569. doi:10.1016/j.ophtha.2016.03.032

9. Wang A, Snead MP. Scleral buckling - a brief historical overview and current indications. Graefes Arch Clin Exp Ophthalmol. 2019;258(3):467-478. doi:10.1007/s00417-019-04562-1

10. Lincoff H, Kreissig I. Advantages of radial buckling. Am $J$ Ophthalmol. 1975;79(6):955-957. doi:10.1016/0002-9394(75) 90677-7

11. Smiddy WE, Loupe DN, Michels RG, Enger C, Glaser BM, Debustros S. Refractive changes after scleral buckling surgery. Arch Ophthalmol. 1989;107(10):1469-1471. doi:10.1001/archopht.198 9.01070020543036

12. Sato EA, Shinoda K, Inoue M, Ohtake Y, Kimura I. Reduced choroidal blood flow can induce visual field defect in open angle glaucoma patients without intraocular pressure elevation following encircling scleral buckling. Retina. 2008;28(3):493-497. doi:10.1097/IAE.0b013e318154ba21

13. Schulze-Bonsel K, Feltgen N, Burau H, Hansen L, Bach M. Visual acuities "hand motion" and "counting fingers" can be quantified with the Freiburg visual acuity test. Invest Ophthalmol Vis Sci. 2006;47 (3):1236-1240. doi:10.1167/iovs.05-0981

14. Talcott KE, Obeid A, Gao X, et al. Pars plana vitrectomy alone for primary rhegmatogenous retinal detachments associated with inferior breaks in phakic eyes. Ophthalmic Surg Lasers Imaging Retina. 2019;50:153-158. doi:10.3928/23258160-20190301-04

15. Sharma A, Grigoropoulos V, Williamson TH. Management of primary rhegmatogenous retinal detachment with inferior breaks. $\mathrm{Br}$ J Ophthalmol. 2004;88:1372-1375. doi:10.1136/bjo.2003.041350
Clinical Ophthalmology

\section{Publish your work in this journal}

Clinical Ophthalmology is an international, peer-reviewed journal covering all subspecialties within ophthalmology. Key topics include: Optometry; Visual science; Pharmacology and drug therapy in eye diseases; Basic Sciences; Primary and Secondary eye care; Patient Safety and Quality of Care Improvements. This journal is indexed on PubMed
Dovepress

Central and CAS, and is the official journal of The Society of Clinical Ophthalmology (SCO). The manuscript management system is completely online and includes a very quick and fair peer-review system, which is all easy to use. Visit http://www.dovepress.com/ testimonials.php to read real quotes from published authors. 